\title{
Swimming Heart: A Pleural, Pulmonary and Pericardial Disseminated Thymoma
}

\author{
Lígia Fernandes*, André Rodrigues, Vera Clérigo, Cláudia Rocha, Dora Sargento, \\ Glória Silva \\ Serviço de Medicina III, Hospital Pulido Valente, Centro Hospitalar Lisboa Norte, Lisboa, Portugal \\ Email: igiasofiafernandes@gmail.com
}

Received 16 September 2014; revised 16 October 2014; accepted 5 November 2014

Copyright (C) 2014 by authors and Scientific Research Publishing Inc.

This work is licensed under the Creative Commons Attribution International License (CC BY).

http://creativecommons.org/licenses/by/4.0/

\begin{abstract}
Thymoma is the most common neoplasm of the anterior mediastinum and accounts for one quarter of all mediastinal tumours and half of all anterior mediastinal masses. Pericardial effusion may be present in approximately $20 \%$ of cases. This report presents a patient with a previously diagnosed thymoma without surgical indication and with poor response to chemotherapy and radiotherapy who had a pericardial effusion that likely resulted from the malignancy dissemination to pericardium.
\end{abstract}

Keywords

Thymoma, Metastases, Pericardial Effusion, Myasthenia Gravis

\section{Introduction}

Although its incidence is rare, thymoma is the most common neoplasm of the anterior mediastinum, accounting to $20 \%$ - $25 \%$ of mediastinal tumours and approximately half of the tumours of the anterior mediastinum, of which 20\% - 30\% are invasive. Most patients are between 40 and 60 years of age, and there is a slight female predominance. Most of thymomas are solid tumours, but more than one third may have necrotic, hemorrhagic or cystic components. Approximately one third of thymomas invade the capsule, extending up to adjacent structures. Transdiaphragmatic extension to the abdomen and pleural and pericardial metastases can occur, however haematogenous and lymphatic dissemination is rare [1].

Since the majority of patients do not present nodal disease or metastases, the tumour, node, metastasis staging

${ }^{*}$ Corresponding author.

How to cite this paper: Fernandes, L., Rodrigues, A., Clérigo, V., Rocha, C., Sargento, D. and Silva, G. (2014) Swimming Heart: A Pleural, Pulmonary and Pericardial Disseminated Thymoma. Open Journal of Clinical Diagnostics, 4, 212-216.

http://dx.doi.org/10.4236/ojcd.2014.44030 
system is not particularly useful for thymomas. Therefore, the extent of the primary tumour is particularly relevant. The Masaoka staging system is a widely applied tool in studies of thymoma, given the tumour rarity and histopathologic heterogeneity [2]. The Masaoka Clinical Staging (Table 1) stratifies thymoma into four groups: non-invasive, confined to the anterior mediastinum tumours, without invasion of neighbouring organs, for stages I and II, and invasive tumours showing invasion of adjacent organs and metastases, for stages III and IV [3].

One third to one half of patients with a thymoma is asymptomatic, and 33\% of patients present with local symptoms related to the involvement of surrounding structures. Although only one-third of patients with localized disease is symptomatic, most patients with disseminated disease have significant complaints, such as chest pain, chest discomfort, dyspnea, and superior vena cava syndrome [4]-[8]. Pericardial involvement by primary mediastinal thymomas often is a late stage finding [9]. Pericardial effusion may be present in approximately $20 \%$ of patients [4]. Thymomas may present with a paraneoplastic syndrome in half of the cases. Therefore $40 \%$ to $45 \%$ of patients present with myasthenia gravis, which is the most common parathymic condition. Despite a generally indolent behaviour, all thymoma are malignant in nature and surgical resection is the pillar of treatment [1] [10]. Complete resection of the entire thymus with (for high-risk tumours) or without neoadjuvant or adjuvant therapy is advised for Stage I and II thymomas. Stage IIIA thymomas should be treated with surgery either initially or after neoadjuvant therapy and the treatment of stage IIIB thymomas may include a combination of chemotherapy, radiation and/or surgery. For Stage IVA tumours, consideration should be given to surgery only if metastases can be resected. The treatment of Stage IVB thymomas is decided on an individual case basis. Noteworthy, chemoradiation should be considered in all medically inoperable patients [2]. Thymomas are usually extremely radiosensitive, and chemotherapy and radiotherapy are usually reserved for locally invasive metastatic disease or inoperable tumours [1] [10]. Even after complete resection, thymomas have a tendency for late recurrence. For recurrent disease, it should be considered surgery, radiation, and/or chemoradiation [2].

The authors report a case of previously diagnosed thymoma associated with pericardial effusion, in a patient with myasthenia gravis, poor response to chemotherapy and radiotherapy and subsequently pericardial disseminated neoplasm.

\section{Clinical Summary}

A 65-year-old caucasian woman, independent in her daily life activities, was admitted in our Internal Medicine Ward with hypercapnic respiratory failure requiring non-invasive ventilation. This patient presented a past medical history of type B2 cortical thymoma with pleural and pulmonary dissemination diagnosed in 2007, not amenable to complete resection, followed by Thoracic Surgery. The chemotherapy performed (8 cycles) had low chemosensitivity and provoked severe toxicity. A diagnosis of parathymic syndrome with myasthenia gravis was confirmed in 2012 and mestinon ${ }^{\circledR}$ therapy was initiated. The latest follow-up computed tomography scans (ten months before the current hospital admission) showed an increased cardiothoracic ratio with scarce pericardial effusion. Physical examination revealed dyspnoea at rest, blood pressure 105/62 mmHg, tachycardia (112 beats/min), with soft heart sounds, but no murmurs or friction rub were auscultated. The neck veins were not distended. Laboratory findings identified increased inflammatory parameters (leukocytosis $12760 \times 10^{6} \mathrm{~L}$, neu-

Table 1. Masaoka clinical staging system of thymoma.

\begin{tabular}{cc}
\hline Stage & Diagnostic criteria \\
\hline I & Macro- and microscopically completely encapsulated tumour \\
II-A & Microscopic transcapsular invasion \\
II-B & Macroscopic invasion into surrounding fatty tissue or \\
III-A & Mrossly adherent \\
III-B & Without invasion of great vessels \\
IV-A & With invasion of great vessels \\
IV-B & Pleural or pericardial dissemination \\
\hline
\end{tabular}

Adapted from [2]. 
trophilia 80\% and elevated CRP $11.5 \mathrm{mg} / \mathrm{dL}$ ). Electrocardiogram presented with sinus tachycardia and low voltage. Chest radiograph revealed an increased cardiothoracic ratio, mediastinal widening and opacity in the middle lobe of the right lung (Figure 1).

A transthoracic echocardiogram was performed which demonstrated a circumferential pericardial effusion with a swimming heart, without diastolic collapse of the right cavities.

Thoracic computed tomography (CT) scans showed a heterogeneous anterior mediastinal lesion with areas of central necrosis, necrotic nodular lesion of the pleura in relation to the right costophrenic angle and significant pericardial effusion. It also presented with extensive areas of lung consolidation with air bronchogram compatible with bilateral basal pneumonia and associated parapneumonic left pleural effusion (Figure 2).

The case was discussed with Thoracic Surgery and Cardiology that excluded the need for pericardial drainage. It was admitted this clinical presentation in the context of a disseminated thymoma to pericardium. The conservative treatment included diuretic therapy with spironolactone and furosemide. Echocardiography was repeated three times, always without signs of cardiac tamponade. The patient fulfilled non-invasive ventilation with gradual weaning and no indication for long-term oxygen therapy. She was discharged clinically improved. Thoracic computed tomography was repeated within 6 months, revealing an increase in the anterior mediastinum mass and metastasis dimensions. New chemotherapy scheme was proposed, however it was discontinued due to patient's intolerance. Currently, the patient has supportive care and monitoring in Thoracic Surgery and Internal Medicine appointments.

\section{Discussion}

Thymomas are typically indolent, slow-growing tumours with local dissemination [2] [10]. Metastases from thymoma typically arise within the thorax, namely pleura, lung and pericardium. Not surprisingly, the malignancy degree of thymomas is more dependable to the extent of the tumour to the thymus capsule and surrounding structures [1]. More advanced thymoma present with symptoms related to the involvement of local structures [2]. Thus, the grade of invasion predicts more accurately the biological potential and behaviour of a thymoma than its histology [3].

According to the Masaoka Clinical Staging, the grade of invasion of our patient's thymoma can be stratified in stage IVA due to its pleural and pericardial dissemination. It was not considered amenable to complete resection so the diagnosis was established prior to therapy using biopsy. Noteworthy, on average, only $25 \%$ of patients with stage IV thymomas are submitted to complete resection. For initially Masaoka stage IVA unresectable disease, it is recommended treatment with neoadjuvant chemotherapy [10]. The thymoma of our case report was submitted to eight cycles of chemotherapy with the intent to improve the chances of being completed resected. The success of treatment was compromised by severe toxicity and low chemosensitivity. Refractoriness to at least one prior chemotherapy regimen is not uncommon in disseminated thymomas. Nonetheless, the availability of data related to the outcomes of chemotherapy as the primary therapy in advanced thymoma is li-

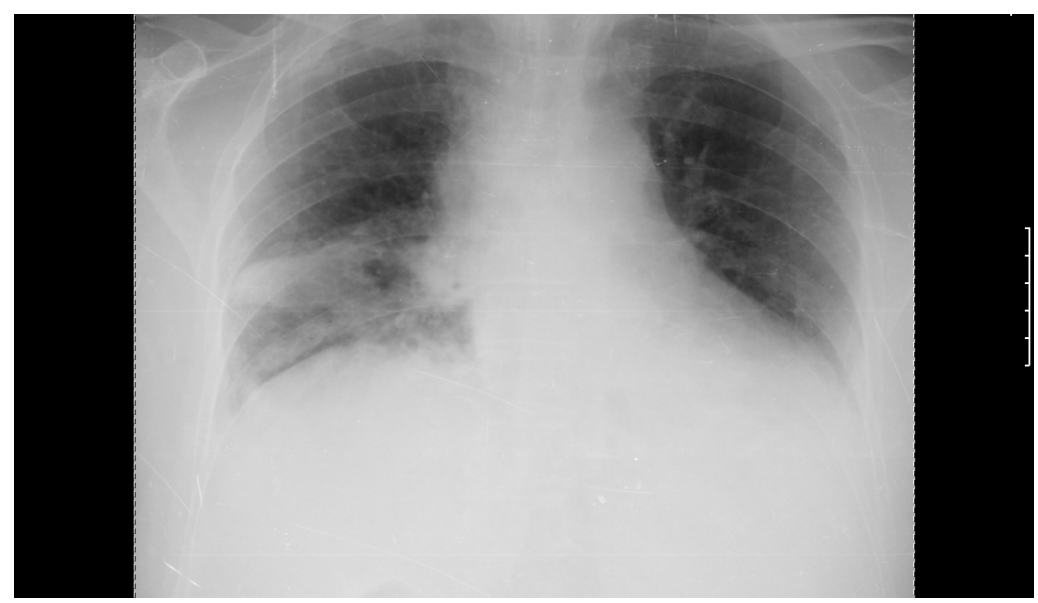

Figure 1. Chest radiograph showed increased cardiothoracic ratio ( $>0.5)$, mediastinal widening, hypotransparent opacity in the middle right pulmonary lobe with dimensions of $41 \times 28 \mathrm{~mm}$ and elevation of the right diaphragmatic dome. 


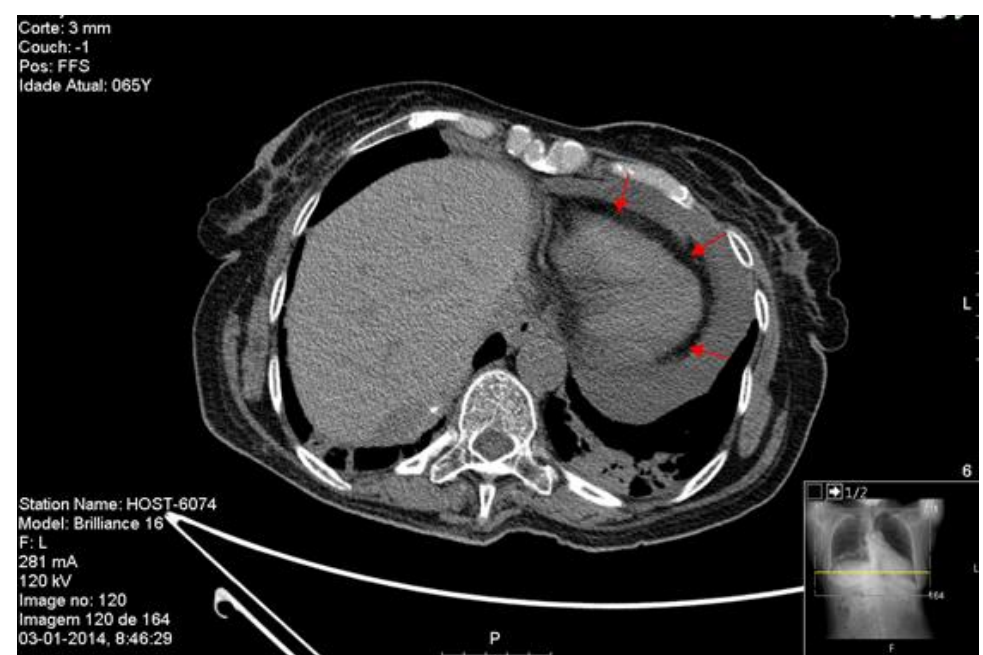

(a)

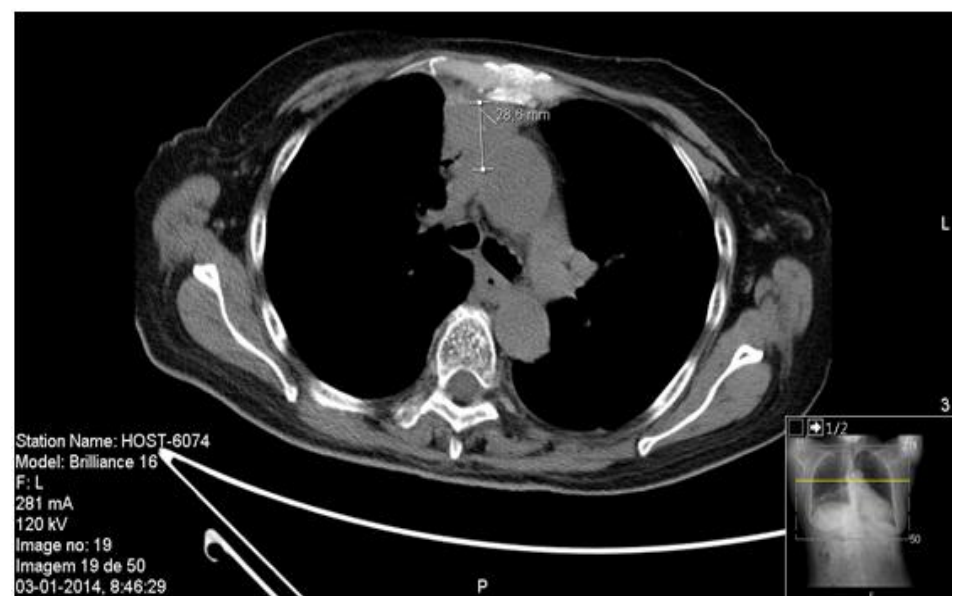

(b)

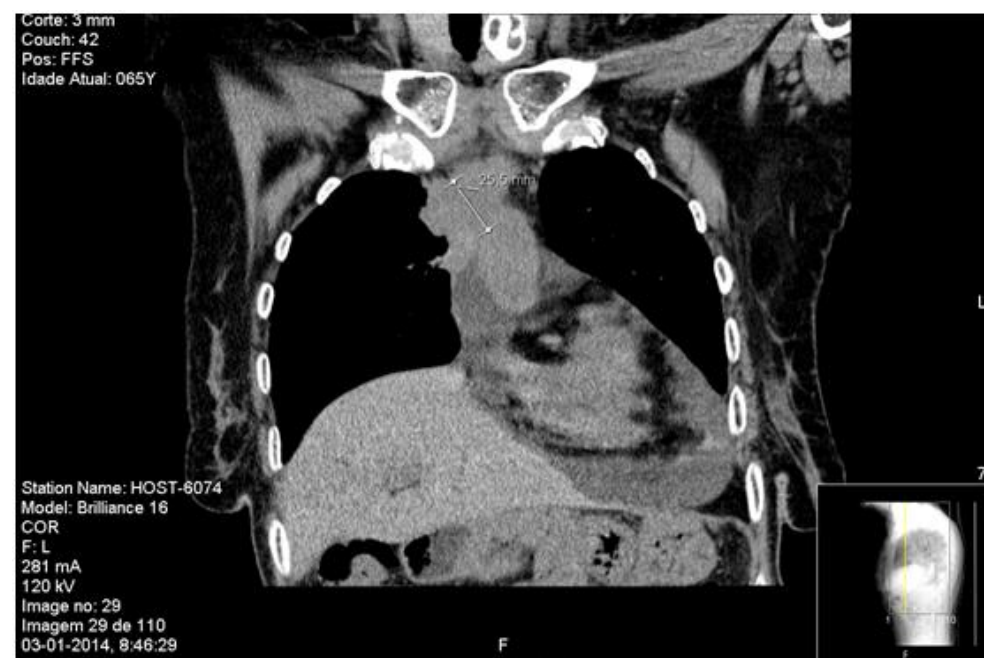

(c)

Figure 2. (a) Thoracic computed tomography revealed significant pericardial effusion (arrows); (b) (c) Thoracic computed tomography showed a nodular mass measuring $28.6 \times 25.5 \mathrm{~mm}$, with irregular borders in the anterior mediastinum, heterogeneous density, areas of coarse calcification and central areas of necrosis at its lower strand located in the right cardiophrenic angle. 
mited [2]. Radiotherapy could provide symptom palliation but our patient was not considered clinically suitable.

Finally, the natural history and prognosis without treatment of thymomas is not yet fully understood. In general, $50 \%$ of the deaths are attributed to the thymoma or to the treatment. Approximately $75 \%$ of deaths in patients with stage IV disease are due to thymoma [10].

\section{Conclusion}

The relative infrequency of thymoma makes this malignancy often poorly understood. Although thymomas are usually slow-growing tumours, the clinical staging is the mainstay for prognostication. Compared to the histological categorization, the Masaoka staging system is a more useful tool when determining the optimal therapy for a thymoma. This case report highlights the need to continue to debate controversial topics such as the choice, sequencing and timing of the neoadjuvant therapies and the appropriateness of surgery in disseminated thymomas.

\section{References}

[1] Almeida, A.B., Hasmucrai, D., Gonçalves, J.R. and Sotto-Mayor, R. (2010) Pneumologia Clínica, 4, 336-366.

[2] Falkson, C.B., Bezjak, A., Darling, G., et al. (2009) The Management of Thymoma: A Systematic Review and Practice Guideline. Journal of Thoracic Oncology, 4, 911-919. http://dx.doi.org/10.1097/JTO.0b013e3181a4b8e0

[3] Suster, S. (2006) Diagnosis of Thymoma. Journal of Clinical Pathology, 59, 1238-1244. http://dx.doi.org/10.1136/jcp.2005.031138

[4] Fazlığulları, O., Atalan, N., Gürer, O., Akgün, S. and Arsan, S. (2012) Cardiac Tamponade from a Giant Thymoma: Case Report. Journal of Cardiothoracic Surgery, 7, 1-4. http://dx.doi.org/10.1186/1749-8090-7-14

[5] Cheng, M.F., Tsai, C.S., Chiang, P.C. and Lee, H.S. (2005) Cardiac Tamponade as Manifestation of Advanced Thymic Carcinoma. Heart Lung, 34, 136-141. http://dx.doi.org/10.1016/j.hrtlng.2004.06.009

[6] Takanami, I., Takeuchi, K. and Naruke, M. (1999) Noninvasive Large Thymoma with a Natural History of TwentyOne Years. The Journal of Thoracic and Cardiovascular Surgery, 118, 1134-1135. http://dx.doi.org/10.1016/S0022-5223(99)70120-0

[7] Soilleux, E.J. (2010) Surgical Pathology of the Thymus and Mediastinum. Diagnostic Histopathology, 16, $143-160$. http://dx.doi.org/10.1016/j.mpdhp.2009.12.009

[8] Wright, C.D. and Kessler, K.A. (2005) Surgical Treatment of Thymic Tumors. Seminars in Thoracic and Cardiovascular Surgery, 17, 20-26. http://dx.doi.org/10.1053/j.semtcvs.2004.12.001

[9] Turkmen, N., Eren, B., Comunoglu, N., Fedakar, R. and Senel, B. (2008) Letter to the Editor-Pericardial Thymoma: An Unusual Cause of Sudden Death. Annals Academy of Medicine, 37, 253-254.

[10] Detterbeck, F.C. (2010) Malignancies of the Thymus-Evaluation and Treatment of Stage I and II Thymoma. Journal of Thoracic Oncology, 5, 318-322. http://dx.doi.org/10.1097/JTO.0b013e3181f20dab 
Scientific Research Publishing (SCIRP) is one of the largest Open Access journal publishers. It is currently publishing more than 200 open access, online, peer-reviewed journals covering a wide range of academic disciplines. SCIRP serves the worldwide academic communities and contributes to the progress and application of science with its publication.

Other selected journals from SCIRP are listed as below. Submit your manuscript to us via either submit@scirp.org or Online Submission Portal.
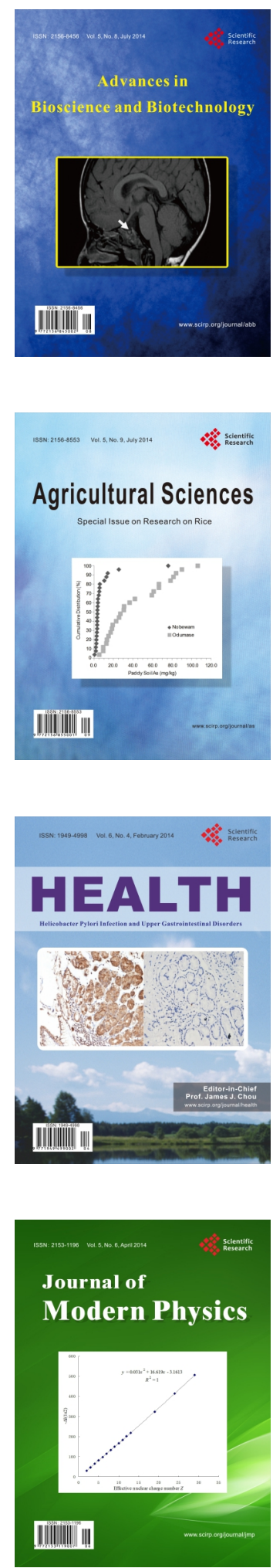
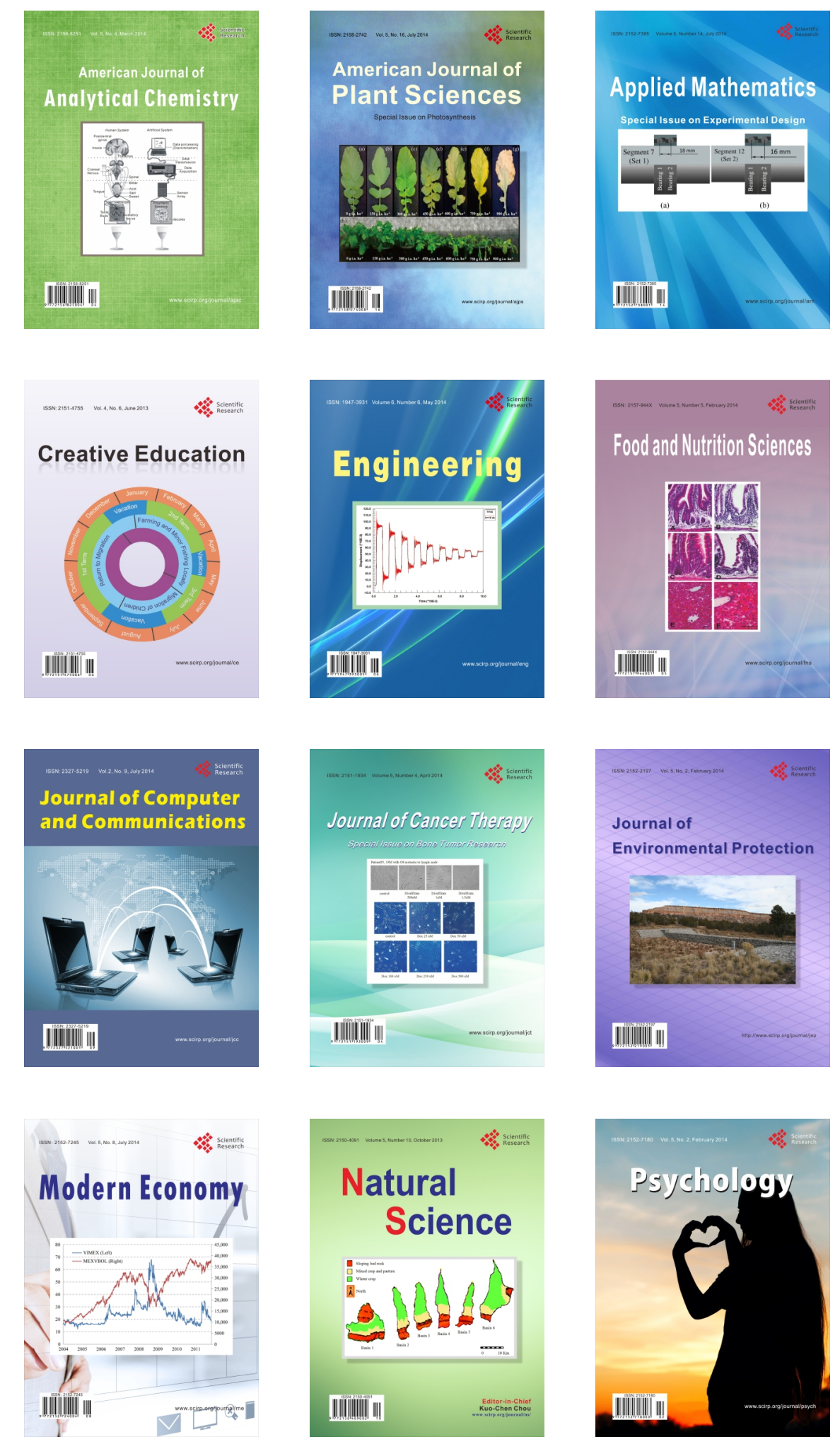